\title{
Do supplemental perches influence electrocution risk for diurnal raptors?
}

\author{
Roberto Sánchez ${ }^{1,2}$, Javier Sánchez ${ }^{2}$, Javier Oria ${ }^{3}$ and Francisco Guil ${ }^{4^{*}}$ (i)
}

\begin{abstract}
Background: Power lines are amongst the main causes of mortality for birds globally. Electrocution drives the population dynamics of several threatened species of raptors, at local and global scales. Among the many solutions that have been tested to minimize this threat are supplemental perches; however, their efficiency has rarely been assessed.

Methods: We designed 43 transects in 4 districts in mainland Portugal to gather data from birds perching on pylons with or without supplemental perches. From 2015 to 2018, transects were surveyed by car at least once. We analyzed the factors driving the use of these supplemental perches, and we analyzed if there were differences in the perceived risk (calculated from measurements and not from field surveys) depending on whether the perching was on pylons with or without supplemental perches.
\end{abstract}

Results: We recorded 548 perches of 14 species. Weather conditions seemed to play a role in birds' choice of pylons with supplemental perches versus pylons without supplemental perches. Models also indicated a strong influence of observational conditions. The use of models showed us an important specific effect in the selection of supplemental perches where available: there are some species with a greater tendency to perch on supplemental perches, even when they use both pylons with and without supplemental perches. For most of the analyzed species and species groups, perceived risk was higher in pylons without supplemental perches compared to pylons with supplemental perches, but there were differences between species.

Conclusions: Supplemental perches may be a useful and efficient tool for mitigating raptor electrocution. However, there are many influential factors affecting their success, and their effectiveness with different species groups is not homogeneous. Some studies show higher electrocution rates for certain species and devices and thus, their efficacy must be validated. We propose a two-step validation process, first in controlled conditions and then in the field. A common protocol should be established to enable comparisons between studies.

Keywords: Aquila adalberti, Circaetus gallicus, Diurnal raptors, Electrocution, Power lines, Pylons

\section{Background}

There is no debate that human development impacts the environment (Griggs et al. 2013). Some of the most heavily studied impacts are those derived from linear infrastructures on biodiversity. A reduction in mammal and bird population densities has been found with increasing

\footnotetext{
*Correspondence: fguil@miteco.es

${ }^{4}$ Ministry for the Ecological Transition and the Demographic Challenge, Plaza San Juan de la Cruz, S/N, 28071 Madrid, Spain

Full list of author information is available at the end of the article
}

proximity to infrastructure (Benítez-López et al. 2010). While there are common elements, the types of impacts vary with the different types of infrastructure such as railroads (Carpenter 1994), roads (Trombulak and Frissell 2000) and power lines (Biasotto and Kindel 2018).

The environmental impacts of power lines are wellknown, despite their wide range. During operation, they mainly impact the landscape, nature, and biodiversity (Bagli et al. 2011). Among the most studied impacts are those related to biodiversity, such as collisions (Bevanger 1998; Bernardino et al. 2018) or electrocution 
(Hernández-Lambraño et al. 2018), but there are other significant impacts, like entanglement (Gangoso and Palacios 2002), effects on reproduction (Fernie and Reynolds 2005) or even an increase in non-native species (Kurek et al. 2015). There are also positive impacts, including the use of pylons by birds as perching (Tomé et al. 2011) or nesting sites (Tryjanowski et al. 2014; Mainwaring 2015).

The main technique to address the negative impacts is adequate planning, including environmental impact assessment (Bagli et al. 2011; D’Amico et al. 2018). However, there are many power lines that are currently impacting the environment. Thus, several ecological restoration techniques to minimize the impacts of existing power lines have been developed, mainly focused on biodiversity (APLIC 2006). However, not all of these techniques appear to be equally effective (i.e. Brown and Drewien 1995; Janss and Ferrer 1999).

Electrocution of birds on power lines greatly impacts the conservation of raptors (Accipitriformes and Falconiformes, hereafter diurnal raptors) in many parts of the world, affecting many species (Janss 2000). It is the main mortality cause for the most endangered raptors, Aquila adalberti and Aquila fasciata (Real et al. 2001; González et al. 2007). The severity of the impact depends on many aspects, such as bird size (Janss 2000), the surrounding habitat (Mañosa 2001), pylon technical characteristics (Hernández-Lambraño et al. 2018), food availability (Guil et al. 2011), and spatial location (Pérez-García et al. 2011).

Techniques to minimize electrocution mainly involve modifying the pylon's technical characteristics, a process known as retrofitting (Chevallier et al. 2015). There are many ways to retrofit a pylon or power pole. The most effective methods involve structural modifications (Tintó et al. 2010), but these are also the most expensive techniques. Isolation can be more moderate in terms of cost and effectiveness (Lehmann et al. 2010), and other practices, such as installing supplemental perches (Dwyer et al. 2016a) or perching deterrents (also called anti-perching devices, discouragers, diverters or guards; APLIC 2006; Dwyer and Doloughan 2014; Dwyer et al. 2016b), have been used.

Supplemental perches and perching deterrents both aim to prevent perching by birds near the conductors. Supplemental perches are usually installed on top of the pylon (i.e. Dwyer et al. 2016a), while perching deterrents are located over the crossarm (with several variations depending on type). Despite their wide use (Karyakin and Barabashin 2005; APLIC 2006), the effectiveness of supplemental perches and perching deterrents has rarely been assessed, and most studies have mainly involved wooden-pole lines (Harness and Garrett 1999; Dwyer and Doloughan 2014, but see Dixon et al. 2019). To our knowledge, there are no studies evaluating the effectiveness of supplemental perches on pylons with pin type insulators.

Pylons with pin type insulators were the most common type of pylon in the Iberian Peninsula, until it was determined that they were a primary source of humaninduced mortality for most threatened raptors (Negro et al. 1989). Since then, many studies have been devoted to this topic (Guil et al. 2011; Ferrer 2012) and great effort has been made to minimize raptor electrocution in general, with particular attention to these threatened species (Tintó et al. 2010; Guil et al. 2015). This has included the increased use of supplemental perches and perching deterrents (Janss and Ferrer 1999). Thus, we consider the Iberian Peninsula a good case study for evaluating the efficiency of supplemental perches in minimizing diurnal raptor electrocution rates.

As a part of the effort to reduce power-line related mortality in Portugal, newly built power lines include supplemental perches on pylons, which are also installed as a retrofitting measure. We sought to determine whether these measures are reducing or augmenting the risk of raptor electrocution, and which factors are driving the use of these pylons by birds.

We chose to analyze several aspects of raptor perches observed during regular fieldwork in four areas of mainland Portugal. In this area, there is only one electricity distributor (EDP), using one primary model for holder and anchor pylons: three-level anchor pylons, partially equipped with a set of supplemental perches, installed for minimizing electrocution risk. These supplemental perches include a pair of inverted V-shaped steel bars that are installed at the top of the pylon, for preventing bird perching on the crossarms (see Fig. 1).

Our goals were to determine if we had an adequate sample of the breeding birds, which factors drive the use of pylons with supplemental perches (compared to pylons without supplemental perches), whether those factors affect all groups of diurnal raptors similarly, and whether there are differences in the perceived risk for diurnal raptors between pylons with and without supplemental perches.

\section{Methods \\ Data gathering}

Data were collected mainly from Dec-2015 to Aug-2018. We added 10 previous data points from large eagles perches in the same area from 2012 to 2014. Data were mainly gathered during the fieldwork carried out as part of the LIFE + Imperial project (http://lifeimperial.lpn.pt/ en). The fieldwork covered vast areas, and thus we only considered data gathered in four districts (Beja, Évora, 


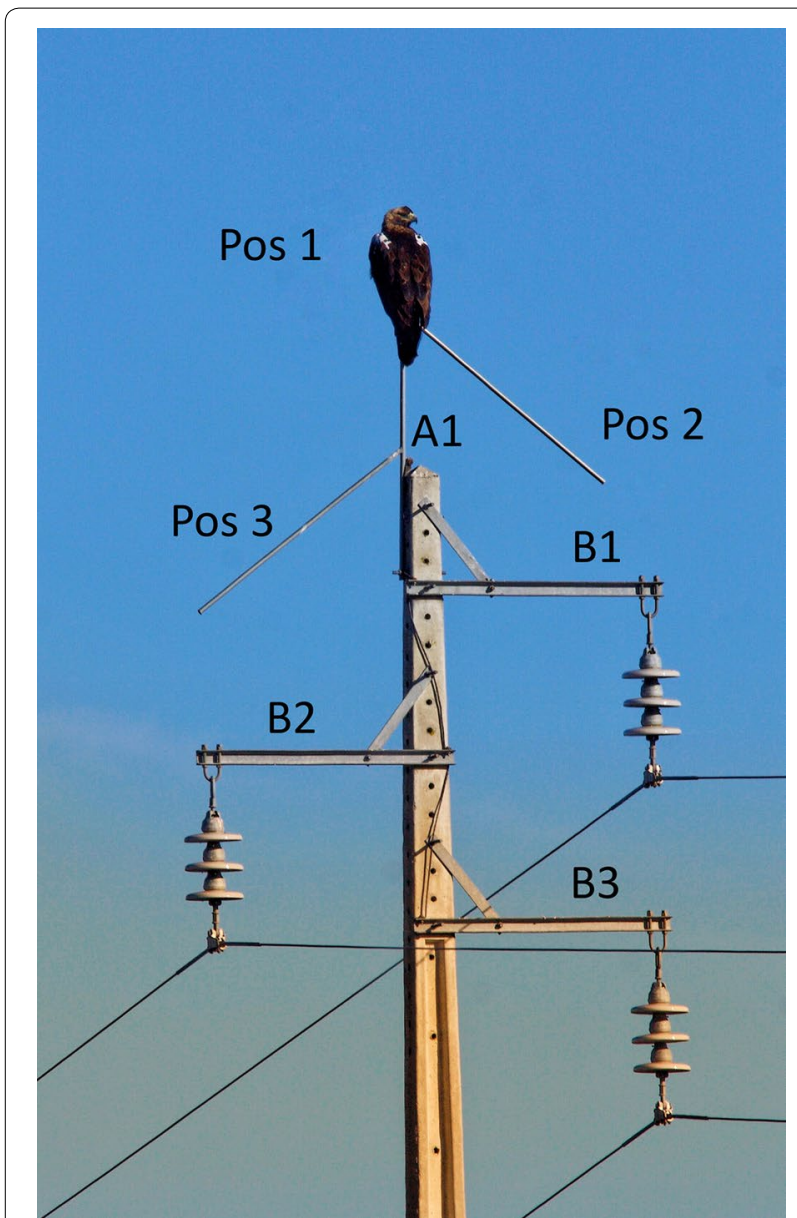

Fig. 1 Three-level anchor pylons with the defined positions (the Spanish Imperial Eagle Aquila adalberti in this photo is perched on position Pos 1 and this is a GAL design)

Portalegre and Castelo Branco), where we could define 43 transects of variable length (see Fig. 2). Transects were designed based on the most frequented areas during the LIFE project. They were not consistently assessed, but were surveyed at least once per month. Transects were surveyed by car, and the driver was the only occupant. We added eight observations from the same region and the same time frame, but collected outside of the transects.

During the transects, data from each perched raptor on three-level pylons were recorded. They included both holder and anchor pylons, being holder pylons those only used to support the conductors and separate them from the ground and anchor pylons those using horizontal insulator strains to generate a mechanical tension to the conductor. Some of these pylons were equipped with a set of supplemental perches. We recorded the species (and, if distinguishable, age and sex), the time of day, the approximate location (the nearest village), and where the bird was perched on the pylon. We defined seven possible perching positions (see Fig. 1):

- A1: At the top of the supporting pylon.

- B1: At the highest point of the crossarm.

- B2: At mid-level of the crossarm.

- B3: At the lowest point of the crossarm.

- Pos 1: At the top of the highest supplemental perch.

- Pos 2: On the highest supplemental perch, not including the top.

- Pos 3: On the lowest supplemental perch.

To control the number of pylons with and without supplemental perches, we surveyed 52 power lines using Google Street View (Google 2020). Those power lines were distributed amongst all of the areas. We surveyed 1018 pylons with a three level crossarm, of which 516 had supplemental perches (50.7\%). Raw data are provided in Additional file 1 .

We gathered weather data through World Weather Online (https://www.worldweatheronline.com/). We requested the nearest temperature (in ${ }^{\circ} \mathrm{C}$ ), humidity (in $\%$, wind $(\mathrm{km} / \mathrm{h})$ and rainfall $(\mathrm{mm} / \mathrm{h})$ in a 3-h lapse per observation and site. We also gathered the geographical coordinates (latitude and longitude) for the center of each locality using Google Earth (Google 2019), with $10 \mathrm{~km}$ as the highest eye elevation. To determine if the birds were within a protected area, we used the World Database on Protected Areas (Protected Planet 2019). We gathered land use data through Corine Land Cover 2018 (Büttner et al. 2017). We considered a $1 \mathrm{~km}$ area surrounding each site covered by forest (cover classes 244 and 31), shrub (cover classes 322 and 323), pastures (cover classes 321 and 333) and annual crops (cover classes 21 and 241). We defined four a priori functional groups of diurnal raptors based mainly on species size (partially after Soldatini et al. 2011 and García 2013, 2017; see Table 1): Vultures, Large eagles, Medium-sized raptors, and Small raptors.

\section{Comparing perching on pylons with and without supplemental perches}

For each functional group and species with a minimum number of perches (more than 10, in both pylons with and without supplemental perches), we analyzed whether there were differences in the usage of each position related to weather conditions (temperature $\left({ }^{\circ} \mathrm{C}\right)$, humidity $(\%)$, wind $(\mathrm{km} / \mathrm{h})$ and rainfall $\left.(\mathrm{mm} / \mathrm{h})\right)$. We also analyzed the time of day when perches were detected. For this purpose, we considered the number of hours since noon (12:00 h). Normality was tested with a Shapiro test. For normally-distributed variables, we used a paired $t$ test and for non-normal 


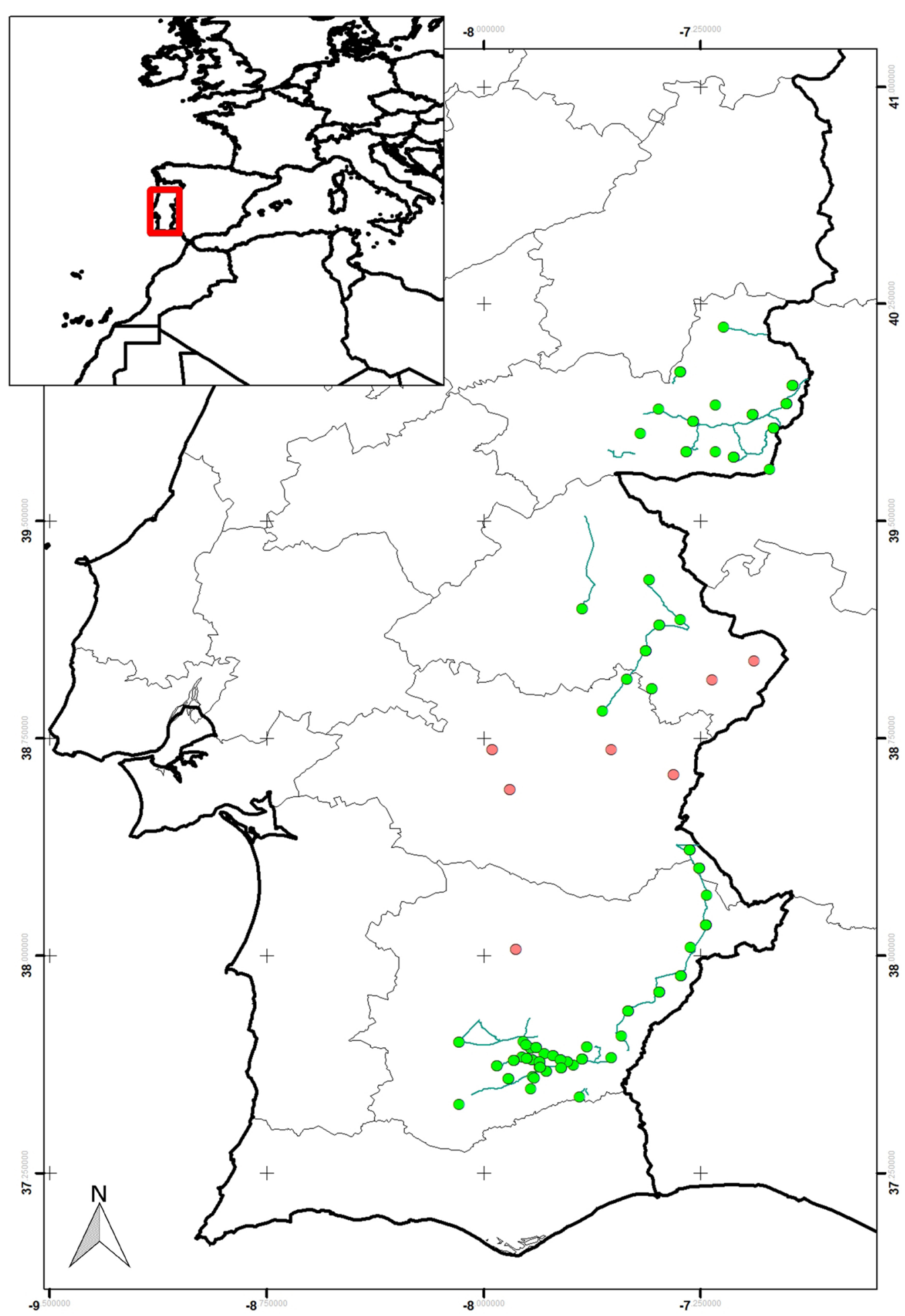

Fig. 2 Surveyed transects (blue lines), observations inside (green dots) and outside (red dots) of the transects

variables, we used a paired Wilcoxon test. We repeated this method to analyze if there were differences in the usage of positions B1 and B2 between pylons with and without supplemental perches. All analyses considered a significance level of 0.05 and were carried out in $R$ 3.5 ( $\mathrm{R}$ Core Team 2019). 
Table 1 Per functional groups and species, number of perches on pylons without (noted as w/o) supplemental perches, perchings on positions B1, B2 and B3 for both pylons, pylons with supplemental perches and, amongst them the number of perchings within the supplemental perches, and years when data were gathered

\begin{tabular}{|c|c|c|c|c|c|c|c|}
\hline \multirow[t]{2}{*}{ Functional group } & \multirow[t]{2}{*}{ Species } & \multicolumn{2}{|c|}{ W/O supplemental perches } & \multicolumn{3}{|c|}{ With supplemental perches } & \multirow[t]{2}{*}{ Years } \\
\hline & & General & B1, B2, B3 & General & B1, B2, B3 & In supps. & \\
\hline Vultures & Gyps fulvus & $17(10 / 2)$ & $4,1,0$ & 0 & 0 & 0 & 2016-2018 \\
\hline \multirow[t]{4}{*}{ Large eagles } & Aquila adalberti & $16(6 / 10)$ & $6,2,0$ & $3(2 / 1)$ & $1,0,0$ & 2 & 2012-2018 \\
\hline & Aquila fasciata & 0 & 0 & $4(3 / 1)$ & $2,1,1$ & 0 & 2015-2017 \\
\hline & Circaetus gallicus & $54(51 / 2)$ & $16,2,0$ & $59(40 / 18)$ & $10,0,0$ & 47 & 2014-2018 \\
\hline & Pandion haliaetus & $3(1 / 2)$ & $1,0,0$ & 0 & 0 & 0 & 2013-2014 \\
\hline \multirow[t]{5}{*}{ Medium-sized raptors } & Hieraaetus pennatus & 1 & $1,0,0$ & 1 & $1,0,0$ & 0 & 2017-2018 \\
\hline & Falco peregrinus & 0 & 0 & 1 & $1,0,0$ & 0 & 2018 \\
\hline & Milvus migrans & 4 & $2,1,0$ & 2 & $0,0,0$ & 2 & 2016-2018 \\
\hline & Milvus milvus & 3 & $2,0,0$ & 1 & $0,0,0$ & 1 & 2018 \\
\hline & Buteo buteo & 104 & $47,17,2$ & 128 & $45,18,8$ & 52 & 2015-2018 \\
\hline \multirow{4}{*}{ Small raptors } & Elanus caeruleus & 20 & 0 & 36 & $1,0,0$ & 34 & 2015-2018 \\
\hline & Falco naumanni & 0 & 0 & 6 & $4,2,0$ & 0 & 2015-2018 \\
\hline & Falco vespertinus & 0 & 0 & 1 & 0 & 1 & 2015 \\
\hline & Falco tinnunculus & 39 & $16,11,3$ & 45 & $19,11,6$ & 3 & 2015-2018 \\
\hline
\end{tabular}

In the functional groups of Vultures and Large Eagles, parentheses indicate the number of perches for adults/juveniles and subadults. Individuals with unknown age are not shown

We also assessed our ability to predict the use of a pylon with versus without supplemental perches. The dependent variables were binary: we considered whether the raptor was perched on a pylon with (1) or without (0) supplemental perches. We contrasted the following six models per analysis using an Information Theoretic approach (Burnham and Anderson 2002): the null model, a model using observation conditions [year (with 5 levels), season (with 4 levels), district (with 4 levels), latitude, and longitude], another using weather variables (temperature, humidity, wind, and rainfall), one with land use [considering the existence of protected areas (with 2 levels) and the land use according to CLC: forest, shrubland, pastures, annual crops], one with the perching species, nested within functional group, and one including all factors. In this approach, the Akaike's Information Criterion (AIC) is typically used as a measure of parsimony (Burnham and Anderson 2002). Hence, we ranked the six models according to their AIC and considered the model with the lowest AIC as the best model explaining the variation in the response variables (Burnham and Anderson 2002). For each model, we used a logistic regression with a log-link function and we gathered Pseudo $R^{2}$ using the DescTools extension (al Asem 2019).

\section{Quantifying perch locations on pylons with supplemental perches}

We used an analogous approach to that used for predicting the use of a pylon with versus without supplemental perches. For those perches occurring in pylons with supplemental perches, we considered whether the raptor was perched on a supplemental perch (1) or not (0). We used the same six models and we ranked them according to their AIC and considered the model with the lowest AIC as the best model explaining the variation in the response variables (Burnham and Anderson 2002). For each model, we used a logistic regression, with a log-link function and we gathered Pseudo $R^{2}$ using the DescTools extension (al Asem 2019).

\section{Perceived electrocution risk on pylons with or without supplemental perches}

For evaluating the variations in the perceived risk, the perches of raptors in different contexts were considered and qualified between 0 and 10, according to these criteria and partially after Guil et al. (unpublished):

10: Perches in position B3 for species with a larger body length (maximum height) than the separation 
of the crossarm and the upper conductor

9: Perches in position B3 for species in which the sum of body (maximum height) and $50 \%$ of the wing length is larger than the separation of the crossarm and the upper conductor

8: Perches in position B3 for species in which the sum of body (maximum height) and the wing length is larger than the separation of the crossarm and the upper conductor

7: Perches in position B3 for species in which the sum of body (maximum height) and the wing length plus $15 \mathrm{~cm}$ is larger than the separation of the crossarm and the upper conductor $(15 \mathrm{~cm}$ is a minimum distance for standard power lines according to the generic method described in INSHT 2011)

6: $\quad$ Other perches in B3

5: Perches in B2 and B1 for species in which the wing length is larger than the insulator strain

4: $\quad$ Perches in B2 and B1 for species in which the wing length is larger than the insulator strain plus $15 \mathrm{~cm}$ (INSHT 2011)

3: $\quad$ Other perches in B2 and B1

2: $\quad$ Perches in A1

1: $\quad$ Perches in Pos 2 or Pos 3

0: $\quad$ Perches in Pos 1

For gathering the body length (maximum height), we used del Hoyo et al. (1994) and for the wing length we used bibliographic references and the CRBPO online database (2020). See Additional file 2. There are two main designs for three-level holder pylons in Portugal: GAL (with approximately $70 \mathrm{~cm}$ between crossarm $\mathrm{B} 3$ and the upper conductor) and GAL1 (with approximately $130 \mathrm{~cm}$ between crossarm B3 and the upper conductor), and average insulator strains were $65 \mathrm{~cm}$ (EDP 2018). As we did not record the type of pylon, we used a probabilistic approach. We analyzed a portion of the holder pylons surveyed to determine the proportion of pylons of GAL and GAL1 designs (Additional file 1), and results showed that $71.2 \%$ had a GAL design and $28.8 \%$ a GAL1. We then calculated the estimated risk in both designs and averaged it using the proportion of pylon designs.

With these perceived risks, for every species or functional group with at least three perches on pylons with and without supplemental perches, we used a sign-rank Wilcoxon test to determine if there were differences, as values were non-normally distributed. As immatures are the most electrocution-prone age group (González et al. 2007), we complimented this analysis with a paired sign-rank Wilcoxon test for differences in the frequency of use of supplemental perches when compared to the use of pylons with supplemental perches for immature individuals.

\section{Results}

We recorded 14 raptor species perching on three-level pylons, for a total number of 548 observations. We considered them to be included in four functional groups. Of those 14 species, only 6 showed a relevant number of perches (more than 10, in both pylons with and without supplemental perches): Gyps fulvus, Aquila adalberti, Circaetus gallicus, Buteo buteo, Elanus caeruleus and Falco tinnunculus.

\section{Comparing perches on pylons with and without supplemental perches}

When analyzing whether there were differences in the usage of each position related to weather conditions, there is an ample range of situations (Table 2). For each functional group and the species with a relevant number of perches (more than 10, in both pylons with and without supplemental perches), the main differences were based on wind conditions, which tended to be higher for perches on pylons with supplemental perches, and significantly higher for large eagles ( $W=1782, p=0.0296)$, Circaetus gallicus $(W=1168.5$, $p$-value $=0.0145)$, medium-sized raptors $(W=6174.5$, $p=0.0158)$, Buteo buteo $(W=5753.5, p=0.0364)$, small raptors $(W=1886.5, p=0.0066)$, and $F$. tinnunculus $(W=514.5, p=0.0011)$. In all cases, the average temperature was higher for perches in pylons without supplemental perches compared to the perches on pylons with supplemental perches, and significantly higher for small raptors ( $W=3168, p=0.0163)$ and $F$. tinnunculus $(W=1154.5, p=0.0129)$.

There were significative differences of use of $\mathrm{B} 1$ and B2 locations between pylons with and without perching supplements $(t=3.09, \mathrm{df}=6, p=0.021)$. For this analysis, as shown in Table 1, we used Large eagles, Medium-sized raptors, Small raptors, Circaetus gallicus, Buteo buteo, Elanus caerulus and Falco tinnunculus perches. As shown in Table 3, the results of the competing models for explaining the number of perches on each type of pylon showed that the whole model was the best model to explain all the aspects, while there was a relevant influence of the observation conditions.

\section{Quantifying perch locations on pylons with supplemental perches}

Amongst the perches on pylons with supplemental perches, the use of models showed us an important specific effect in the selection of supplemental perches where available: there are some species with a greater 
Table 2 Climatic parameters for species and functional groups with a relevant number of perches, for perches on pylons with and without (noted as w/o) supplements

\begin{tabular}{lrrrrr}
\hline & Temp. & Humidity & \multicolumn{1}{c}{ Wind } & Rainfall & Hour \\
\hline Large eagles w/o supplements & $24.46 \pm 8.77$ & $42.01 \pm 20.28$ & $\mathbf{9 . 3} \pm \mathbf{5 . 1 2}$ & $0.09 \pm 0.28$ & $3.89 \pm 2.61$ \\
Large eagles with supplements & $24.92 \pm 7.70$ & $46.6 \pm 21.54$ & $\mathbf{1 0 . 9 5} \pm \mathbf{4 . 2 2}$ & $0.05 \pm 0.20$ & $3.71 \pm 2.36$ \\
Circaetus gallicus w/o supplements & $25.93 \pm 7.86$ & $39.65 \pm 19.53$ & $\mathbf{8 . 8 3} \pm \mathbf{4 . 6 2}$ & $0.06 \pm 0.16$ & $4.10 \pm 2.62$ \\
Circaetus gallicus with supplements & $25.92 \pm 7.29$ & $44.59 \pm 21.31$ & $\mathbf{1 1 . 0 9} \pm \mathbf{4 . 1 0}$ & $0.05 \pm 0.21$ & $3.81 \pm 2.42$ \\
Aquila adalberti w/o supplements & $19.5 \pm 10.1$ & $50 \pm 21.36$ & $10.88 \pm 6.48$ & $0.23 \pm 0.48$ & $3.16 \pm 2.51$ \\
Aquila adalberti with supplements & $13 \pm 4.58$ & $63 \pm 11.53$ & $6 \pm 2$ & $0 \pm 0$ & $2.88 \pm 1.69$ \\
Medium-sized raptors w/o supplements & $20.78 \pm 8.18$ & $54.69 \pm 21.24$ & $\mathbf{1 0 . 0 8} \pm \mathbf{7 . 3 6}$ & $0.03 \pm 0.11$ & $3.48 \pm 1.92$ \\
Medium-sized raptors with supplements & $19.75 \pm 6.73$ & $55.44 \pm 23.09$ & $\mathbf{1 1} \pm \mathbf{5 . 0 4}$ & $0.09 \pm 0.35$ & $3.65 \pm 1.87$ \\
Buteo buteo w/o supplements & $20.87 \pm 8.41$ & $54.46 \pm 21.73$ & $\mathbf{1 0 . 2 8} \pm \mathbf{7 . 4 7}$ & $0.04 \pm 0.11$ & $3.38 \pm 1.9$ \\
Buteo buteo with supplements & $19.75 \pm 6.66$ & $54.95 \pm 22.91$ & $\mathbf{1 0 . 9 5} \pm \mathbf{4 . 9 7}$ & $0.08 \pm 0.35$ & $3.64 \pm 1.87$ \\
Small raptors w/o supplements & $\mathbf{2 3 . 2 4} \pm \mathbf{6 . 9 0}$ & $50.59 \pm 21.92$ & $\mathbf{8 . 4 2} \pm \mathbf{4 . 8 9}$ & $0.06 \pm 0.19$ & $4.28 \pm 1.96$ \\
Small raptors with supplements & $\mathbf{2 0 . 4 7} \pm \mathbf{7 . 6 5}$ & $51.23 \pm 23.06$ & $\mathbf{1 1 . 4 2} \pm \mathbf{7 . 1 0}$ & $0.12 \pm 0.36$ & $3.78 \pm 2.18$ \\
Falco tinnunculus w/o supplements & $\mathbf{2 3 . 2 6 \pm 6 . 2 4}$ & $48.51 \pm 21.28$ & $\mathbf{8 . 4 7} \pm \mathbf{4 . 6 1}$ & $0.07 \pm 0.22$ & $4.21 \pm 2.07$ \\
Falco tinnunculus with supplements & $\mathbf{1 9 . 9 1} \pm 6.80$ & $52.51 \pm 21.73$ & $\mathbf{1 2 . 5 8} \pm \mathbf{7 . 2 2}$ & $0.19 \pm 0.47$ & $3.54 \pm 2.21$ \\
Elanus caeruleus w/o supplements & $23.2 \pm 8.21$ & $54.65 \pm 23.13$ & $8.4 \pm 5.52$ & $0.04 \pm 0.11$ & $4.42 \pm 1.79$ \\
Elanus caeruleus with supplements & $18.86 \pm 7.04$ & $55.06 \pm 22.68$ & $9.33 \pm 6.96$ & $0.04 \pm 0.16$ & $3.98 \pm 1.98$ \\
\hline
\end{tabular}

Italicized show normal distribution of both parameters and a $t$ test

Bolditalics show significant differences

Table 3 Results of the competing models for comparing perches on pylons with and without supplemental perches, where AIC is akaike information criterion, $\triangle \mathrm{AIC}$ the difference with the most parsimonious model and McFadden's Pseudo $R^{2}$

\begin{tabular}{llcl}
\hline Model & AIC & AAIC & Pseudo $\boldsymbol{R}^{\mathbf{2}}$ \\
\hline Whole & 377.30 & 0 & $60.20 \%$ \\
$\begin{array}{l}\text { Observation condi- } \\
\quad \text { tions }\end{array}$ & 412.88 & 35.58 & $49.42 \%$ \\
Land use & 653.57 & 276.27 & $15.51 \%$ \\
Climatic & 711.92 & 334.62 & $10.97 \%$ \\
Species & 725.38 & 348.08 & $8.14 \%$ \\
Null & 758.97 & 381.67 & 0 \\
\hline
\end{tabular}

Table 4 Results of the competing models for comparing perch locations on pylons with supplemental perches, where AIC is akaike information criterion, $\triangle A I C$ the difference with the most parsimonious model and McFadden's Pseudo $R^{2}$

\begin{tabular}{llll}
\hline Model & AIC & DAIC & Pseudo $\boldsymbol{R}^{\mathbf{2}}$ \\
\hline Climatic & 295.47 & 0 & $34.28 \%$ \\
Species & 299.83 & 4.36 & $31.17 \%$ \\
Whole & 300.52 & 5.05 & $42.56 \%$ \\
Null & 399.82 & 104.35 & 0 \\
Land use & 403.81 & 108.34 & $2.02 \%$ \\
Observation condi- & 407.63 & 112.16 & $4.58 \%$ \\
$\quad$ tions & & & \\
\hline
\end{tabular}

tendency to perch on supplemental perches, even when they use both pylons with and without supplemental perches (see Tables 1 and 4). There is also a strong influence caused by climatic conditions.

\section{Perceived electrocution risk on pylons with or without supplemental perches}

The results for the perceived risks for every species or functional group with at least three perches on both pylons with and without supplemental perches show that, for most of the analyses, perceived risk was lower for perches on pylons with supplemental perches compared to those without them. Results are shown in Table 5.

The analysis of the frequency of juveniles and immatures perching on supplemental perches compared to the frequency of juveniles and immatures perching on pylons with supplemental perches revealed no differences ( $W=5, p=0.4142$ ). For this analysis, as shown in Table 1, we used Circaetus gallicus, Aquila adalberti and Aquila fasciata perches.

\section{Discussion}

Our results show that supplemental perches may be a useful and efficient tool for mitigating raptor electrocution, but there are many factors influencing their use by electric companies. 
Table 5 Average perceived risk for observed perchings on pylons with or without (noted as w/o) supplemental perches for every species or functional group with at least three perchings on pylons with and without supplemental perches; also shown are results from the Wilcoxon test and $p$-values for those analyses

\begin{tabular}{lccll}
\hline Group/species & Risk w/o suppl. & Risk with suppl. & Wilcoxon test & $\boldsymbol{p}$-value \\
\hline Small raptors & $2.718 \pm 1.068$ & $1.905 \pm 1.933$ & $W=1997$ & 0.016 \\
Elanus caerulus & $2.05 \pm 0.224$ & $0.139 \pm 0.593$ & $W=29$ & $3.824 e^{-11}$ \\
Falco tinnunculus & $3.06 \pm 1.167$ & $3.171 \pm 1.634$ & $W=927$ & 0.594 \\
Medium-sized raptors & $2.72 \pm 1.0885$ & $2.036 \pm 2.094$ & $W=5751.5$ & 0.001 \\
Buteo buteo & $2.721 \pm 0.907$ & $2.053 \pm 2.108$ & $W=5268$ & 0.001 \\
Large eagles & $2.726 \pm 0.961$ & $1.004 \pm 1.87$ & $W=829$ & $1.837 e^{-11}$ \\
Circaetus gallicus & $2.667 \pm 0.952$ & $0.78 \pm 1.521$ & $W=486$ & $1.325 e^{-11}$ \\
Aquila adalberti & $3 \pm 1.033$ & $1.333 \pm 2.309$ & $W=12$ & 0.156 \\
\hline
\end{tabular}

Italics show significant differences

\section{Comparing perching on pylons with and without supplemental perches}

Despite our results show a strong specific component, there are also common aspects. For instance, all groups and species of birds tend to perch on pylons with supplemental perches on windier and colder days. As perches on pylons with supplemental perches tend to occur more during midday, the use of these pylons could be related to hunting behavior, with the use of pylons without supplemental perches related to resting behavior, as well as territoriality and temperature. Our study did not reveal clear patterns, and thus more work is needed to disentangle these differences. However, what is clear is that supplemental perches must be carefully designed, given that perchings on supplemental perches occur on windier days. This could also be related to their greater stability and greater ease for supplemental perches compared to trees. While the differences are not significant, average humidity was higher for perchings on supplemental perches for every analyzed group and species. Materials and shapes (especially grip characteristics, diameter and section) should be assessed when designing supplemental perches (i.e. Dwyer et al. 2016a). A correct design is essential for a long-lived structure (Dwyer et al. 2020).

The most parsimonious model for explaining the use of pylons with or without supplemental perches included observation conditions (year, season, district, latitude, and longitude). This model was able to explain a relevant amount of variability, and only the complete model explained more. There may be a number of reasons underlying this finding, but the absence of alternative perches could be key, as this partially explains the habitat differences. This aspect might also be related with the differential presence of supplemental perches in protected areas, where the proportion of pylons with supplemental perches is over $88 \%$, compared to the overall $51.9 \%$. These aspects may also be partially represented in the observation conditions (especially in the district and coordinates), which can be also related to the protected areas, as the proportion of observations considered inside protected areas varies highly within districts (from less than $0.5 \%$ in Portalegre district ( 2 out of 243 observations) to $75 \%$ in Beja (33 out of 44 observations)). On the other hand, we might also find spatial differences underlying the absence or scarcity of perches of certain species (i.e. Gyps fulvus or Aquila adalberti). These species were mainly seen in the Castelo Branco district, which has a low percentage of pylons with supplemental perches (approximately $8 \%$, according to our data). A more systematic survey could help disentangle these effects.

\section{Quantifying perch locations on pylons with supplemental perches}

The use of models showed us an important specific effect in the selection of supplemental perches where available: there are some species with a greater tendency to perch on supplemental perches, even when they use both pylons with and without supplemental perches. While Circaetus gallicus and Elanus caerulus seem to perch most often in these dominating positions, others seldomly or never perch on supplemental perches, such as Aquila fasciata and Falco tinnunculus. Those results are aligned with the information shown in Table 1. This could be related to a higher adaptability to variable climatic conditions for migrating raptors (Blanco, pers. com.). Raptors without a feathered tarsus probably have a better thermoregulation system (Mosher and White 1978). There may also be coloration aspects at play, as darker morphs present a lower reflectance and higher temperature excess when irradiated than pale morphs (Roulin 2004), and Circaetus gallicus and Elanus caerulus are paler than Aquila fasciata or Falco tinnunculus. Thus, the climatic and specific variables may be interrelated. 
This could explain the relatively low increase in explained variability in the whole model, compared to the specific and climatic models. However, there could also be territorial aspects, which are discussed later.

There are many individual and specific factors affecting the use of perches (Guil et al. 2015). Our results confirm this tendency for species differences in the use of pylons without and with supplemental perches, and amongst these, the use of the supplemental perches instead of the crossarm. This plays an important role in the variations of the perceived risks, and thus further research is needed to validate these supplemental perches as an efficient tool for a wide variety of diurnal raptors.

\section{Perceived electrocution risk on pylons with or without supplemental perches}

The use of this conservation measure for a broad group of species (diurnal raptors in this case) may benefit some species and be ineffective for others. In some cases, electrocution risk may increase, as shown in Table 5 for Falco tinnunculus. Despite it is a very small species and perceived risks are very low, this should be taken into account as a potential drawback for other species in other situations. Nonetheless, all of the detected risks remain low (less than 2.5 out of 10 in all cases), and especially in some cases such as Elanus caerulus. For most of the birds and functional groups, the perceived risk tends to decline. However, we must be cautious when interpreting these results, as this study only gathered perceived risks, assigned to the observed perching birds, and there are evident limitations, as we had no separated data for holder and anchor pylons. In most of the studies this kind of pylons shows higher electrocution rates than holder ones (Guil et al. 2011).

While we were not able to detect significant differences when comparing frequency of use of supplemental perches to the use of pylons with supplemental perches for immature individuals, the data shown in Table 1 indicates that juveniles of Aquila adalberti and A. fasciata do not perch on supplemental perches. It is possible that adult individuals tend to perch on higher, visible perchings, partly to be more visible, as a territorial remark (Mahaffy and Frenzal 1987; Turrin and Watts 2014). A complementary explanation, based on experience, could be related to supplemental perches being less stable than the crossarm. This could partially explain why immature age-classes are amongst the most vulnerable to electrocution (Real et al. 2001; González et al. 2007). Some changes in the design (materials and dimensions) to make the supplemental perch more stable and, especially, a more suitable perching area in Pos 1 might increase the efficacy of this measure for certain species. A horizontal bar in this position might be useful.
More research should be carried out to confirm these elements as a valid mitigation tool, especially related to juveniles. We propose a two-step validation process. The first step should take place in controlled conditions with different species and functional groups, using the elements of real crossarms (Dwyer et al. 2016a; Guil et al. 2015). This first step could indicate if there are target species rejecting these elements, as occurred here with Gyps fulvus. The observed behavior of this species, as well as other heavy species such as Aegypius monachus, could be related to the design of perches, as they are relatively long and unstable. Thus, they are probably rejected as perching sites despite the considerations of spatial effects. The second, more relevant, step should involve a field survey (as in Dixon et al. 2018 and preferably, with camera traps, as in Dwyer and Doloughan 2014). The design of these studies should consider especially the migrating species, as migrating periods show higher mortality rates (Klassen et al. 2014).

The efficacy of diverters and supplemental perches as a mitigation tool has been widely discussed (Harness and Garret 1999; Janss and Ferrer 1999; Dwyer et al. 2016b; Dixon et al. 2018), but most studies have only focused on the presence of mortality. The effect seems to vary greatly between studies, some of them reporting even higher electrocution rates than on not-retrofitted poles (Pérez-García et al. 2019). We have detected many factors conditioning the use of supplemental perches. There may also be many factors influencing perching deterrents efficiency. Thus, the goal should be to establish a common protocol for evaluating mitigating efficacy, considering the proposed two-step procedure. This may help to disentangle the factors determining successful measures, and to increase coordination between studies. This should also reduce the cost of implementation of efficient measures, and even reduce local extinctions of birds that use these types of structures.

\section{Conclusions}

Our work suggests, in line with previous studies (Janss and Ferrer 1999; Dixon et al. 2019), that supplemental perches reduce electrocution risk. However, the results are not homogeneous among species. In addition, some of the species that seem to reject their use are highly affected by electrocution (Real et al. 2001). If temperature is a factor in limiting the use of pylon supplemental perches, as our results suggest, the use of supplemental perches as a generic measure in warm areas could be ineffective for a wide range of species. With the increase in temperatures as a result of climate change (Trigo and Palutikof 1999), this measure could become ineffective for many species in the study area. 
In the case of the Spanish Imperial Eagle, it has been shown that electrocution mortality has a greater impact on the non-breeding population compared to the reproductive (territorial) individuals (González et al. 2007). The supplement designs analyzed in this study would be more effective in breeding areas where there is a risk of mortality for breeding individuals. It would be useful to design supplemental perches that are accepted by both breeding and juvenile (non-breeding) birds.

There are many factors conditioning the use of supplemental perches. Thus, the goal should be to establish a common protocol for evaluating the mitigating efficacy as a practical solution for diverse species.

\section{Supplementary information}

Supplementary information accompanies this paper at https://doi. org/10.1186/s40657-020-00206-9.

Additional file 1. Power lines.

Additional file 2. Measurements.

Additional file 3. Raw data.

\section{Acknowledgements}

Field work for this project was conducted under the LIFE + Nature project "Conservation of the Spanish Imperial Eagle (Aquila adalberti) in Portugal"LIFE13 NAT/PT/001300, supported 75\% by the Life Programme-European Commission. We are indebted to Ángel Arredondo, Carlos Molinero, Juan Manuel Pérez-García, Marisa Pérez and Roberto Carbonell for their collaboration in the initial versions of the manuscript. Comments from three anonymous referees seriously improved the manuscript.

\section{Authors' contributions}

$\mathrm{RS}$ and JO conceived and designed the experiments. RS and JS conducted the field work. FG performed the data analysis and wrote the early draft, and RS and $\mathrm{JO}$ revised and improved the manuscript. All authors read and approved the final manuscript.

\section{Funding}

Field work for this project was conducted under the LIFE + Nature project "Conservation of the Spanish Imperial Eagle (Aquila adalberti) in Portugal" LIFE13 NAT/PT/001300, supported 75\% by the Life Programme-European Commission.

\section{Availability of data and materials}

All data generated or analyzed during this study are included in this published article and its Additional file 3.

\section{Ethics approval and consent to participate}

The experiments comply with the current laws of Portugal. RS had a permit for conducting the fieldwork from the Portuguese authorities.

\section{Consent for publication}

Not applicable.

\section{Competing interests}

The authors declare that they have no competing interests. The funders had no role in study design, data collection or analysis, decision to publish, or preparation of the manuscript. And none of the authors was hired or contracted by EDP or any other Portuguese power company.

\footnotetext{
Author details

1 Fundación CBD-Hábitat, C/Gustavo Fernández Balbuena, 28002 Madrid, Spain. ${ }^{2}$ Tragsa Group, C/Conde de Peñalver, 84, 28006 Madrid, Spain. ${ }^{3}$ Boscaje
}

S.L., Plaza Conde de Cheste, 5, 40001 Segovia, Spain. ${ }^{4}$ Ministry for the Ecological Transition and the Demographic Challenge, Plaza San Juan de la Cruz, S/N, 28071 Madrid, Spain.

Received: 21 December 2019 Accepted: 22 June 2020

Published online: 26 June 2020

\section{References}

Al ASem. DescTools: Tools for descriptive statistics. R package version 0.99.29. 2019. https://cran.r-project.org/package=DescTools. Accessed 15 Sep 2019.

APLIC (Avian Power Line Interaction Committee). Suggested practices for avian protection on power lines: the state of the art in 2006. Sacramento: Edison Electric Institute, Washington, DC, Avian Power Line, Interaction Committee, and the California Energy Commission; 2006.

Bagli S, Geneletti D, Orsi F. Routeing of power lines through least-cost path analysis and multicriteria evaluation to minimise environmental impacts. Environ Imp Asses. 2011;31:234-9.

Benítez-López A, Alkemade R, Verweij PA. The impacts of roads and other infrastructure on mammal and bird populations: a meta-analysis. Biol Conserv. 2010;143:1307-16.

Bernardino J, Bevanger K, Barrientos R, Dwyer JF, Marques AT, Martins RC, et al. Bird collisions with power lines: state of the art and priority areas for research. Biol Conserv. 2018:222:1-13.

Bevanger K. Biological and conservation aspects of bird mortality caused by electricity power lines: a review. Biol Conserv. 1998;86:67-76.

Biasotto LD, Kindel A. Power lines and impacts on biodiversity: a systematic review. Environ Imp Asses. 2018:71:110-9.

Brown WM, Drewien RC. Evaluation of two power line markers to reduce crane and waterfowl collision mortality. Wildlife Soc B. 1995;23:217-27.

Burnham KP, Anderson DR. Model selection and multimodel inference: a practical information-theoretic approach. 2nd ed. Berlin: Springer; 2002.

Büttner G, Kosztra B, Soukup T, Sousa A, Langanke T. CLC2018 technical guidelines. Copenhagen: EEA; 2017.

Carpenter TG. The environmental impact of railways. Chichester: Wiley and Sons; 1994.

Chevallier C, Hernández-Matías A, Real J, Vincent-Martin N, Ravayrol A, Besnard A. Retrofitting of power lines effectively reduces mortality by electrocution in large birds: an example with the endangered Bonelli's eagle. J App Ecol. 2015;52:1465-73.

D'Amico M, Catry I, Martins RC, Ascensão F, Barrientos R, Moreira F. Bird on the wire: landscape planning considering costs and benefits for bird populations coexisting with power lines. Ambio. 2018;47:1-7.

Del Hoyo J, Elliott A, Sargatal J. Handbook of the birds of the world. New world vultures to guineafowl, vol. 2. Barcelona: Lynx Edicions; 1994.

Dixon A, Bold B, Tsolmonjav P, Galtbalt B, Batbayar N. Efficacy of a mitigation method to reduce raptor electrocution at an electricity distribution line in Mongolia. Conserv Evid. 2018;15:50-3.

Dixon A, Rahman ML, Galtbalt B, Bold B, Davaasuren B, Batbayar N, et al. Mitigation techniques to reduce avian electrocution rates. Wildlife Soc B. 2019;43:476-83.

Dwyer JF, Doloughan KW. Testing systems of avian perch deterrents on electric power distribution poles in sage-brush habitat. Hum-Wild Interact. 2014:8:39-55.

Dwyer JF, Taylor RC, French GA. Failure of utility pole perch deterrents modified during installation. J Raptor Res. 2020;54:172-6.

Dwyer JF, Tincher MC, Harness RE, Kratz GE. Testing a supplemental perch designed to prevent raptor electrocution on electric power poles. NW Nat. 2016a;97:1-7.

Dwyer JF, Tincher MC, Harness RE, Kratz GE. Successful use of a perch deterrent to manipulate raptor perching on model power poles. Color Birds. 2016b;50:166-74.

EDP. Desenhos técnicos normativos. armações de aço para postes de betão de MT. Lisboa: EDP Distribuçao; 2018.

Fernie KJ, Reynolds SJ. The effects of electromagnetic fields from power lines on avian reproductive biology and physiology: a review. J Tox Env Heal B. 2005;8:127-40. 
Ferrer M. Birds and power lines. From conflict to solution. Sevilla: ENDESA and Fundación MIGRES; 2012.

García V. Guía de identificación de rapaces ibéricas por restos óseos (1. a parte, grandes rapaces). Madrid: Ministerio de Agricultura, Alimentación y Medio Ambiente; 2013

García V. Guía de identificación de rapaces ibéricas por restos óseos (2. a parte, rapaces medianas diurnas). Madrid: Ministerio de Agricultura y Pesca, Alimentación y Medio Ambiente; 2017.

Gangoso L, Palacios CJ. Endangered Egyptian Vulture (Neophron percnopterus) entangled in a power line ground-wire stabilizer. J Raptor Res. 2002;36:238-9.

González LM, Margalida A, Mañosa S, Sánchez R, Oria J, Molina Jl, et al. Causes and spatio-temporal variations of non-natural mortality in the vulnerable Spanish imperial eagle Aquila adalberti during a recovery period. Oryx. 2007:41:495-502.

Google. Google Earth digital images. Google, Mountain View, CA, USA. Several places. Retrieved November 2019.

Google. Google Streetview digital images, Google Maps (http://maps.googl e.com). Several places. Accessed March 2020. See Additional file 1 for URLS.

Griggs D, Stafford-Smith M, Gaffney O, Rockström J, Öhman MC, Shyamsundar P, et al. Policy: sustainable development goals for people and planet. Nature. 2013:495:305.

Guil F, Fernández-Olalla M, Moreno-Opo R, Mosqueda I, Gómez ME, Aranda A, et al. Minimising mortality in endangered raptors due to power lines: the importance of spatial aggregation to optimize the application of mitigation measures. PLoS ONE. 2011;6:e28212

Guil F, Colomer MÀ, Moreno-Opo R, Margalida A. Space-time trends in Spanish bird electrocution rates from alternative information sources. Global Ecol Conserv. 2015;3:379-88.

Harness RE, Garrett M. Effectiveness of perch guards to prevent raptor electrocutions. J Col Field Ornithol. 1999;33:215-20.

Hernández-Lambraño RE, Sánchez-Agudo JÁ, Carbonell R. Where to start? Development of a spatial tool to prioritise retrofitting of power line poles that are dangerous to raptors. J App Ecol. 2018;55:2685-97.

INSHT. Arco eléctrico: estimación de la energía calorífica incidente sobre un trabajador. Notas Técnicas de Prevención. Madrid: INSHT; 2011.

Janss GFE. Avian mortality from power lines: a morphologic approach of a species-specific mortality. Biol Conserv. 2000;95:353-9.

Janss GFE, Ferrer M. Mitigation of raptor electrocution on steel power poles. Wildlife Soc B. 1999;27:263-73.

Karyakin IV, Barabashin TO. Dark holes in the raptor populations (electrocutions of birds of prey on power lines in the Western Betpak-Dala), Kazakhstan. Raptor Conserv. 2005;4:29-32.

Klaassen RH, Hake M, Strandberg R, Koks BJ, Trierweiler C, Exo KM, et al. When and where does mortality occur in migratory birds? Direct evidence from long-term satellite tracking of raptors. J Anim Ecol. 2014;83:176-84.

Kurek P, Sparks TH, Tryjanowski P. Electricity pylons may be potential foci for the invasion of black cherry Prunus serotina in intensive farmland. Acta Oecol. 2015;62:40-4.
Lehman RN, Savidge JA, Kennedy PL, Harness RE. Raptor electrocution rates for a utility in the intermountain western United States. J Wildlife Manage. 2010;74:459-70.

Mahaffy MS, Frenzel LD. Elicited territorial responses of northern Bald Eagles near active nests. J Wildlife Manage. 1987;51:551-4.

Mainwaring MC. The use of man-made structures as nesting sites by birds: a review of the costs and benefits. J Nat Conserv. 2015;25:17-22.

Mañosa S. Strategies to identify dangerous electricity pylons for birds. Biodivers Conserv. 2001;10:1997-2012

Mosher JA, White CM. Falcon temperature regulation. Auk. 1978;95:80-4.

Negro JJ, Ferrer M, Santos C, Regidor S. Eficacia de dos métodos para prevenir electrocuciones de aves en tendidos eléctricos. Ardeola. 1989;36:201-6.

Pérez-García JM, Botella F, Sánchez-Zapata JA, Moleón M. Conserving outside protected areas: edge effects and avian electrocutions on the periphery of Special Protection Areas. Bird Conserv Int. 2011;21:296-302.

Pérez-García JM, Morales-Reyes Z, Naves-Alegre L, Sánchez-Zapata JA, Sebastián-González J. Mortalidad de aves por electrocución y colisión en líneas eléctricas en el desierto del Gobi. P. 121. Madrid: SEO/BirdLife 2019. Libro de Resúmenes del VII Congreso Ibérico y XXIV Español de Ornitología. https://www.seo.org/wp-content/uploads/2019/11/LIBRO -RESUMENES-2019.pdf. Accessed 5 Dec 2019.

Protected Planet. World database on protected areas. October 2019 Release. UNEP-WCMC-WCPA. 2019. https://www.protectedplanet.net/. Accessed 15 Nov 2019

Real J, Grande JM, Mañosa S, Sánchez-Zapata JA. Causes of death in different areas for Bonelli's Eagle Hieraaetus fasciatus in Spain. Bird Study. 2001:48:221-8.

Roulin A. The evolution, maintenance and adaptive function of genetic colour polymorphism in birds. Biol Rev. 2004;79:815-48.

Soldatini C, Albores-Barajas YV, Lovato T, Andreon A, Torricelli P, Montemaggiori $A$, et al. Wild life strike risk assessment in several Italian airports: lessons from BRI and a new methodology implementation. PLOS ONE. 2011;6:e28920.

Tintó A, Real J, Mañosa S. Predicting and correcting electrocution of birds in Mediterranean areas. J Wildlife Manage. 2010;74:1852-62.

Tomé R, Dias MP, Chumbinho AC, Bloise C. Influence of perch height and vegetation structure on the foraging behaviour of Little Owls Athene noctua: how to achieve the same success in two distinct habitats. Ardea. 2011;99:17-27.

Trigo RM, Palutikof JP. Simulation of daily temperatures for climate change scenarios over Portugal: a neural network model approach. Clim Res. 1999;13:45-59.

Tryjanowski P, Sparks TH, Jerzak L, Rosin ZM, Skórka P. A paradox for conservation: electricity pylons may benefit avian diversity in intensive farmland. Conserv Lett. 2014;7:34-40.

Turrin C, Watts BD. Intraspecific intrusion at Bald Eagle nests. Ardea. 2014;102:71-9.

Trombulak SC, Frissell CA. Review of ecological effects of roads on terrestrial and aquatic communities. Conserv Biol. 2000;14:8-30.
Ready to submit your research? Choose BMC and benefit from:

- fast, convenient online submission

- thorough peer review by experienced researchers in your field

- rapid publication on acceptance

- support for research data, including large and complex data types

- gold Open Access which fosters wider collaboration and increased citations

- maximum visibility for your research: over $100 \mathrm{M}$ website views per year

At BMC, research is always in progress.

Learn more biomedcentral.com/submissions 